\title{
Why do people refuse to take part in biomedical research studies? Evidence from a resource-poor area
}

Joseph Mfutso-Bengo ${ }^{1}$, Francis Masiye ${ }^{1}$, Malcolm Molyneux ${ }^{2}$, Paul Ndebele ${ }^{1}$, Abdullah Chilungo ${ }^{1}$

1. Centre for Bioethics in Eastern and Southern Africa, CEBESA, College of Medicine, Malawi.

2. The Malawi-Liverpool- Wellcome Trust Research Programme, College of Medicine, Blantyre, Malawi

Corresponding author: Francis Masiye, Centre for Bioethics in Eastern and Southern Africa (CEBESA), Department of Community Health, College of Medicine, Private Bag 360, Chichiri, Blantyre 3, Malawi Phone: +265 1871 911, Email: fmasiye@medcol.mw

\section{Abstract}

Participants' refusal to take part in research is an unpleasant experience that investigators face.. This paper highlights some of the reasons why people from resource-poor settings refuse to take part in health research. This paper also highlights standards which investigators can adopt to avoid unnecessary refusals and at the same time ensure that individuals have the right to participate and freedom to refuse. Our objective was to explore reasons why people refuse to join research studies. We conducted focus group discussions with people who had refused to take part in a number of biomedical research studies but agreed to be interviewed in this study. The study was undertaken in the peri-urban and urban areas of Blantyre district; Bangwe, Mpemba and Madziabango. We found nine key factors that influence people to refuse to participate in biomedical research. The factors are failure to follow traditional customs , lack of study benefits, superstition, poor informed consent procedures, ignorance of health research, fear of strangers, lack of cultural sensitivity, poor timing, and previous bad research experience. People refuse to participate in health research for a number of reasons which can be overcome if researchers embark on community engagement before implementing their studies.

\section{Introduction}

Ethical conduct in research involving human participants has become one of the principal challenges faced by researchers. In recent times, increasing attention has been paid to ethical implications of carrying out research in settings with low socio-economic status. This trend has become particularly central in Africa owing to an increasing number of multinational research institutions operating in sub-Saharan African countries. ${ }^{1-4}$

The progress of medicine today is a result of biomedical research which necessarily relies on successful recruitment of human participants in order to yield meaningful results. In this sense, human participants are instrumental in securing data useful to researchers. A participant's refusal to participate in research is referred to as a non-response in a survey. ${ }^{5}$ For the purpose of this paper, we shall use the term 'refuser' to refer to individuals who declined to take part in research after being approached by research staff.

At the heart of ethical recruitment of participants in a study is the principle of informed consent. There is a general consensus amongst researchers, scientists and bioethicists that acquiring effective informed consent from research participants is a prerequisite to the conduct of an ethically sound study ${ }^{6}$. Among other things, valid informed consent requires that participation in research should be voluntary and that individuals should be treated as autonomous agents? The requirement for voluntary participation, however, may result in many people refusing to participate. Refusals are likely to affect the timeline of research and also compromise the generalizability of results, ${ }^{8}$ since it is unclear how people who refuse differ from those who agree to participate. There has been limited empirical research aiming to capture the perspectives of refusers in developing countries.

Many studies have investigated the reasons why people participate in biomedical research ${ }^{9-12}$ however, there has been less research and little attention specifically on reasons why people refuse to participate in biomedical research. Most of the data available on refusals are centred on non response $^{13}$ to postal questionnaires from different surveys.

In this paper we report findings from an anthropological and bioethics study of clinical research in Malawi. The study explored the reasons why people refuse to join biomedical research and examined possible strategies for minimising refusals. The participants that were interviewed in this study had refused to participate in a number of different biomedical research projects before this study but agreed to be interviewed in this study. We believe that knowledge of participants' perspectives and concerns with theirinvolvement in research can lead to better recruitment efforts, improve the informed consent process, and enhance the overall trust between participants and researchers ${ }^{4}$.

Many studies have investigated the reasons why people participate in biomedical research ${ }^{9-12}$ however, there has been less research and little attention specifically on reasons why people refuse to participate in biomedical research. Most of the data available on refusals is centred on non response ${ }^{13}$ to postal questionnaires from different surveys.

This paper reports findings from an Anthropological and Bioethics Study of Clinical Research in Malawi (ABSCRM). The study explored the reasons why people refuse to join biomedical research and examined possible strategies for minimising refusals. The participants that were interviewed in this study had refused to participate in a number of different biomedical research projects before this study but agreed to be interviewed in this study. Knowledge of participants' perspectives and concerns with their involvement in research will enhance recruitment efforts, improve the informed consent process, and enhance the overall trust between participants and researchers ${ }^{4}$.

\section{Methods}

\section{Design}

FFor this study, a qualitative approach was adopted and data were collected through focus group discussions (FGDs). A total of 108 refusers were successfully recruited and they participated in 12 FGDs, each composed of 6-12 people. The FGD questionnaire guide was translated into the local 
language Chichewa.. All FGDs were recorded on audio tapes which were then transcribed and translated from Chichewa into English by members of the research team.

\section{Setting}

The study was conducted in Blantyre District, in the Southern region of Malawi. The specific sites were in the peri-urban settings of Madziabango and Mpemba health centre catchment areas, and Bangwe Township which is a very densely populated urban area. These locations were selected for this project because several medical research projects have been conducted there in the past.

\section{Recruitment}

Refusers were identified by project staff from the registers for the various trials which were ongoing at the time. These registers were maintained at the three sites by the project staff for the various projects. Each project register had details of all the participants involved in the study as well as those who declined to participate after being invited to enrol. Health Surveillance Assistants (HSAs), who are part of the community, were then tasked with the responsibility of following up the refusers in their homes in order to inform them about the current project and to invite them to visit the health centres or other specific locations for the FGDs. Once the individuals had gathered for each FGD our research team ensured that they all met the eligibility criteria for refusers... Verbal consent was obtained from those who agreed to participate in the FGDs. It was difficult to find sufficient refusers willing to take part in the focus group discussions. Therefore some FGDs had to be rescheduled.

\section{Data analysis}

Analysis of the FGD transcripts was carried out manually and electronically using computer software N6*. We analysed the contents of the transcribed texts to identify patterns and themes that emerged from the data. ${ }^{15}$ A comparison of major themes was then made in the final stage of analysis.

\section{Research Ethics Committee Approval}

The study was approved by the College of Medicine Research Ethics Committee (COMREC).

\section{Results}

\section{Demographics}

Overall, there were more women than men in the refusers' FGDs. Of the 108 participants, $21 \%$ were males and $79 \%$ were females.. There were no significant differences in average age between the male participants (31 years) and the female (30 years).. Between the peri-urban and urban respondents, there was a slight difference in the average duration of education -7 and 9 years respectively.

\section{Description of findings}

We identified ten different self-reported reasons why people had refused to take part in various health studies. These were:

- Failure to follow traditional customs

- Fear of strangers

- Superstition

- Poor informed consent procedure
- Lack of study benefits

- Ignorance of health research

- Lack of cultural sensitivity

- Poor timing

- Previous bad research experience

\section{Failure to follow traditional customs}

The overall perception of the participants is that traditional procedure has a big impact in making people decide whether to take part in a study or not. They reported that failure to conduct community engagement and failure to follow the right protocol during recruitment of participants would make people refuse to take part in research. The procedure they suggested as a better practice was for interested researchers to first seek consent from their chief or community leader and then conduct sensitization meetings and a community gathering where the whole community would be briefed about the study. If the chief says yes to a research project to take place in the area, his/her subjects would equally feel secure to accept and take part in it. For example one participant from Mpemba said
"they should first inform the leaders of the community like the chief and when they accept, the people would also accept the research. But if they just come from wherever they come from and start the research without involving our community leaders, people will not accept it because they are not aware."

In response to the question 'why is community engagement important?' several participants self-reported that community engagement would give them a chance to understand more information about the study and be able to ask questions to the researchers before deciding whether to accept or decline the research. They also reported that people refuse to participate in studies when they are taken by surprise and not informed in advance about the study. This leaves the communities unprepared to decide whether to participate or not. One participant said

\section{"some researchers just arrive without informing the chief and the people. We can refuse because we have not been informed."}

Another participant said

"the researchers were coming here all over a sudden.

They would just send a short message that we are coming tomorrow and moreover among the visitors was a white person. It is better maybe if they can start telling the people this week that next week visitors will come."

Participants also argued that another advantage of community engagement is that it would dispel rumours and misconceptions that circulate in their communities.

\section{Fear of strangers}

Participants reported that people refuse to participate in health studies when researchers are strangers in the community. One participant reported

\footnotetext{
"it will be difficult for us to accept their research if they

just come without even wearing a uniform and being people we have never seen them here before."
} 
Another respondent reported

"people in the villages here are afraid to see strangers moving from house to house, everybody would run away because we have never seen such people in our community, for example, there was this group of researchers that was going around the villages with a white man distributing drugs and mosquito nets. For us villagers, we rarely see white people in our homes. So it was difficult to accept the drugs and mosquito nets which they brought to us because we could not trust them."

However, several participants reported that they would be comfortable to associate themselves with ordinary villagers and local doctors they know.

\section{Superstition and blood drawing}

People refuse and express reservations if studies constitute interventions that involve drawing of blood. This is tied to beliefs where by blood is seen as a weapon used in witchcraft for inflicting pain or sending pestilence to people. Belief in and fear of witchcraft is the norm amongst most Malawians. 1617

'In magic, blood is revered and feared for the miraculous power

it possesses and confers. Blood is believed to unleash power.'

Other people also associate the drawing of blood with rumours about blood sucking. ${ }^{18}$ They also fear that more blood would be drawn from their bodies. Several participants emphasized that studies that involve drawing of blood are very risky and have a greater potential for refusal. One participant observed

"most people fear that their blood will be drawn out if they participate in research".

On the other hand, some participants said that they were discouraged to take part in studies that involved taking of blood simply because they were told that they would be given presents if they consented to donate blood. For example, one participant said

"People were discouraged to participate in the study because they heard that participants who gave a bottle of blood in exchange were given basins and wrappers."

Other fears that were reported concerning drawing of blood and dispensation of drugs include drawing more blood for sale, and rumours that drugs being dispensed are intended to make people barren or sterile. Participants said that people see this as a government conspiracy to reduce population growth. For example, one participant said that;

\begin{abstract}
"at first I had refused to participate in the Onchocerciasis study because there were a lot of things being said about it, that the government wanted to make sure that many people are not having many children since many of us are not going for family planning [Participant is talking about distribution of Mectizan drugs]. Many of us got scared with these stories. But when we saw this other lady with skin itches all over her stomach, we saw that what others were saying is not true. So we changed our minds."
\end{abstract}

\section{Poor informed consent procedures}

Participants reported that people refuse to take part in studies when researchers fail to explain clearly about the objectives of their research. One participant said
"Sometimes it also depends on how the researchers explain their research to people. If they do not explain it clearly, people will not understand it and it will be difficult for them to participate."

\section{Lack of study benefits}

People decline to participate in research that does not provide benefits which can either accrue to the individual or to the area as a whole. Participants reported that they would like to participate in studies that would benefit them and not just to join studies only for altruistic reasons. For example, one participant said

"What people expect to hear is how the research would be conducted and how the study will benefit them. If they see that indeed things can improve, they can say yes, let the study take place, mainly when they see that the study will be beneficial in uplifting their health."

\section{Ignorance of health research}

Ignorance and lack of understanding about research are some of the factors that would influence people's refusal to participate in health research. One participant observed

"The other thing that researchers from the urban areas should know when coming here is that they will be meeting people from the village. And we lack understanding in most things. It is important therefore to take time to explain to us and come to an agreement. We should first be told the purpose of the research before starting to conduct it so that we should have time to think about it otherwise if we are rushed we would refuse."

Another participant questioned

"don't researchers know that some of us never went further with school? Instead of explaining properly so that we understand, he sometimes spoke in English and that used to confuse us as to what he really meant".

Analysis of the interviewees' responses also shows that many people especially from the rural areas are ignorant about certain procedures in health research. One participant who refused to take part in a malaria study expressed discontentment over the use of medicated bed-nets. The participant said
"the white man tells me to sleep under a mosquito net that has got medication in it. What if I wake up in the morning only to find sores all over my body?"

Similarly, several participants were not aware of the rationale behind re-taking of blood samples every year especially in longitudinal studies. One participant expressed disappointment and said

"they can get blood today. A year can elapse before coming again and request to take your blood again. You wonder as to what they did with the blood they took the first time. People refuse to participate because of that."

\section{Lack of cultural sensitivity}

Several participants shared the view that they refused to take part in studies where researchers were not respectful to them. Participants observed from a number of studies that 
researchers were either disrespectful or did not know how to approach the people. One participant said

"researchers must talk to the people with love and patience. They ought to know how to approach the people. They should not threaten them. If people are threatened, they will not listen to them. But if they talk to them with love, they would definitely understand."

Participants also noted that researchers overlooked the importance of approaching husbands in studies that targeted women. Researchers did not involve husbands of the women they sampled during the consent seeking process. This resulted in either many women refusing to participate or husbands deterring their wives from taking part. One participant said

\section{"busbands of many women are very troublesome. Women may be shouted by their husbands if they accept to take part in a study right at the hospital. It would have been good if researchers approached married couples in the villages and explained to both of them together"}

Several participants were also dismayed and refused to join a Mectizan drugs studies because researchers were measuring people's heights with a rigid ruler (wooden rod) to determine dosage. Mostly a wooden rod is used to measure the height of a dead body for funeral purposes. For example, one participant said

"on the issue of the rod, the dead are measured with a stick. And they measure you with a stick too. This is not good."

\section{Poor timing}

Participants reported that there are certain periods of the year when they are engaged in their fields with farming activities. These moments are not convenient for rural participants to enrol in research studies. They said that most people refuse to take part during the rainy season when they work in their farms. One participant from Madziabango said

"during the farming period, people are busy. They have no time for research. It is important to know the time people are free to do things like research."

\section{Previous bad research experience}

Participants reported that they would reject studies when they look back at what similar previous studies had done to them. If studies have not been helpful people would refuse to join them. Similarly, they would decline to participate if such studies harmed people's lives. Many people reported to have refused to take part in studies in which HIV testing was involved due to psychological reasons. One participant said

"I refused to participate in the AIDS research because I felt that if they found the virus in my blood I would be a worried person and cannot live peacefully in my home knowing that any day I will die."

\section{Discussion}

Several reasons why people refuse to participate in health research have been revealed in this study. Many of these might also be applicable to refusers in other similar settings.

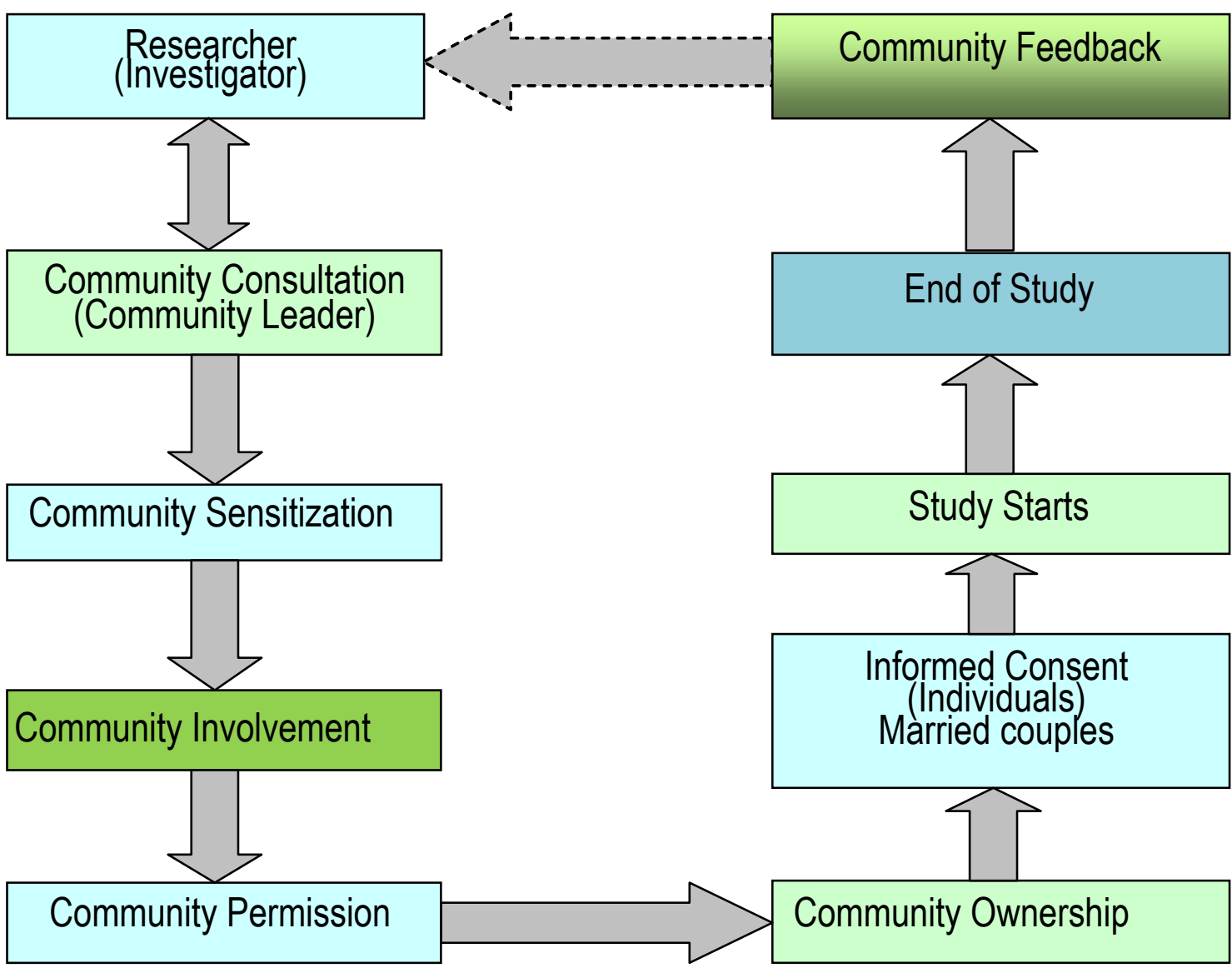


Participants in our sample were those who had previously refused to take part in a number of different biomedical research activities.

Participants in our sample reported that they would refuse to participate in a study where the researchers failed to follow the traditional custom. Figure 1 below illustrates the traditional custom that participants want researchers to follow. Following the traditional custom would benefit the researcher because it would minimise unnecessary refusals. What participants are referring to as traditional custom is in fact community engagement, which we have modified to suit the needs of all participants. This is illustrated in Figure 1.

Community engagement is a process of engaging community members in research through consultation, sensitization, involvement and providing feedback. In Figure 1, the steps for community engagement show a lineal pattern with one entry point for the researcher which is the community leader. Participants agree that the researcher has to go through the community leader. If the community leader accepts the study then he or she should sensitize the community by organising a meeting to involve them in the study. The community involvement meeting should be attended by the researcher, community leader and all the community members. Among other things, during this meeting, the researcher is expected to introduce and brief the community members about the study. Community members should also have a chance to ask the researcher questions where they do not understand. The community can give its permission whether they want the study to be conducted in their community. This also ensures that the community members have ownership of the research project. Informed consent process follows community involvement. Single people can be invited to participate individually while married persons can be interviewed as a couple to facilitate joint decision making. Participants feel that when all this is successfully carried out, then the study can begin.

Participants stressed the importance of observing the community engagement for successful recruitment and informed consent. This demonstrates that people would like to participate in studies if they are satisfied with the rationale and information about the study. There is wide acknowledgement of the need for community engagement in biomedical research, particularly in developing countries. Currently, engaging communities has become a critical aspect of planning and implementing biomedical research. The current international research ethics guidelines also talk of community engagement as an ethical requirement for research involving human subjects, particularly with marginalized populations. ${ }^{19} \quad$ Genuine community engagement offers hope of enhancing recruitment, retention, and participant satisfaction.

In addition to concerns with strangers, participants also expressed discomfort with studies that involved drawing of blood. These concerns can be addressed by adhering to the village protocol in Figure 1. It would dispel any myths about the study. Furthermore, the issue of fearing strangers would not arise when researchers participate in community engagement.

Our results raise an interesting point regarding the informed consent procedure: poor informed consent with vague explanation of objectives will discourage participation. This brings up a long-running debate about how much information about the study should be disclosed to prospective study participants. There is no consensus on this. If we argue that the information should be 'as much as possible' then we beg another question; how much is 'as much as possible?' According to the Belmont report, genuine informed consent requires that participants should be given sufficient comprehensive information and they should be allowed to have enough time for consideration. They should also be offered an opportunity to ask questions. This result shows that the conduct of informed consent remains a big challenge in settings with limited resources. More research is needed about participants' perceptions and experiences in health research in settings with limited resources in order to improve the informed consent processes. However, we feel that adoption of the community engagement procedure would demystify the conduct of informed consent in resource poor settings.

Another important theme that emerged from this study was the importance and application of the principle of beneficence. Participants reported that they would not participate in research studies which do not either benefit them or the community. Participants also reported that some studies set bad precedents which demoralise their future participation in similar studies. Resource poor settings remain potential targets for exploitative research.

Our results showed that people in peri-urban areas would refuse to participate in health research owing to ignorance and lack of understanding about certain concepts and procedures. Researchers need to know the level of understanding about health research of their potential participants. The significance of using community engagement as a way of informing the community about the study should therefore not be underestimated. During community engagement, the researcher can find out if there is need to educate potential participants about certain procedures involved in the study.

Finally, another factor which contributes to people's refusal to take part is the concern that some researchers are not conscious of local culture. Results show that participants were disgruntled with researchers who were not friendly, hospitable and lacked skills to properly approach participants. Several participants from Mpemba and Madziabango said that they were not happy to see researchers measuring heights of people with a rod which is normally used to measure corpses. Researchers should know more about the aspects of culture of the people they would be investigating, and try to anticipate the ways in which their actions and props like measuring rods might be interpreted locally. A number of researchers seem to be in a hurry to implement their studies while neglecting the welfare of their participants. This compromises the ethical conduct of research. Knowing the culture, daily lifestyles, and opinions of participants would help researchers know when and how to best approach their participants.

\section{Recommendations}

The traditional customs suggested by the refusers constitute the first step towards minimising refusals. We believe that adequate community engagement will minimise refusals. 
We offer the following specific recommendations as principles of community engagement which we have developed as a result of this study.

\section{Four Principles of Community Engagement in Research}

\section{Community consultation}

- Brief the community leaders (chiefs, political leaders and religious leaders) about the research project

organize a meeting with them

- introduce the research project to the leaders

- explain in detail all the procedures involved in the research

- outline any risks and benefits to individual participants and to the community

- allow the leaders to ask questions about the research project

- make sure you cover the topics listed in (3) below

- obtain their permission to conduct the research project in their community.

- Alternatively, you may approach the community through meeting with a Community Advisory Board $(\mathrm{CAB})$ where one exists

members of the $\mathrm{CAB}$ can then explain the project to community leaders and seek their permission for the research project to be conducted in their community

When permission has been granted, the researchers, community leaders and members of the $\mathrm{CAB}$ can organize a community sensitization meeting.

\section{Community sensitization}

- organize sensitization workshops for community members

- inform them about the proposed research

- encourage a shared effort between researchers and participants

- ensure that the research activities are responsive to the needs of the community

- make sure that members of the community understand what the research is all about

- define clearly the role that will be played by the community members

- clarify any risks and any compensations

- allow community members to ask questions about the project.

[Important: you should avoid providing information that might lead to stigma and discrimination. For example, if a research project is about HIV/AIDS and one of the recruitment criteria is that potential participants have to be HIV positive, this fact should not be made known to the whole community, as they would then know that people recruited in the study are HIV positive. A common solution to this problem is to design the study so that both HIV+ve and HIV-ve individuals are enrolled, and enrolment then does not imply any individual's HIV status.]

\section{Community Ownership/partnership/involvement}

- Regard local communities participating in research as partners or 'owners'.

- This partnership should begin before the conduct of the research project and should continue during and after the completion of the research project.

- Agree in advance with the community leaders about what will happen after the research is over

- During any study, it is likely that the researchers will need to respond to urgent needs of the community or of individuals. As far as possible, agree in advance with donors, Ethics Committee and community leaders about the possible form and extent of such assistance.

\section{Feedback}

- When results are available, feed these back to research participants and their community.

- Make this effort, even if results may not be of immediate benefit to participants, and despite the fact that it may be difficult to trace all who took part in the study.

- Community feedback is another way of fostering ownership (in this case, ownership of results).

We conducted feedback sessions about the results in our study sites. We wrote a letter to the Clinical Officer-in-charge of each of the health centres, informing them about our schedule for the dissemination of results. We also requested the local HSAs who had helped us with the recruitment of participants to inform all the five categories of people we had recruited. We prepared power point presentation slides together with printed handouts in the local language, Chichewa. About $80 \%$ of the participants were present during the dissemination of results. Participants appreciated this feedback. They reported that this was the first time they had seen researchers disseminating results to the community in which they had conducted their research.

\section{Conclusion}

We believe that most 'refusers' would have been willing to participate in research if the above principles had been carefully followed. Our findings require validation in a larger sample from other settings in Malawi and other developing countries, but meanwhile researchers need to take into account the concerns raised by the refusers we interviewed. Most of these concerns could be minimised by engaging the community, both before research is initiated and after it has been completed.

\section{Acknowledgements}

We would like to express our sincerely thanks and gratitude to The Wellcome Trust for funding this study. 


\section{References}

1. Benatar SR: Reflections and recommendations on research ethics in developing countries. Social Science \& Medicine 2002, 54:1131-1141

2. Zulfiqar AB: Ethics in international health research: a perspective from the developing world. Bull World Health Organ 2002, 80:114-120

3. Shapiro HT, Meslin EM: Ethical issues in the design and conduct of clinical trials in developing countries. N Engl J Med 2001, 345:139142

4. Khalil, S.S. et al. Attitudes, understanding, and concerns regarding medical research amongst Egyptians: A qualitative pilot study. BMC Medical Ethics 2007, 8 (9): 1472-6939

5. McCall, N., Trofimovich, L., and Bonito, A. Estimation and Analysis of Non-Response Bias in Medicare Surveys. RTI International. August 2004

6. Lidz, W.S et al, "Therapeutic misconception and the appreciation of risks in clinical trials. Social Science and Medicine", 58(2004) 16891697, pg 1689

7. The Belmont report. The National Commission for the Protection of Human Subjects of Biomedical and Behavioral Research. April 18, 1979. http://ohsr.od.nih.gov

8. Jones J. The effects of non-response on statistical inference. J Health Soc Policy 1996; 8: 49-62

9. Brody, J. L. \& Waldron, H. B. Ethical issues in research on the treatment of adolescent substance abuse disorders. Journal of Addictive Behaviors 2000; Vol. 25, (2): 217-228. www.emhr.net

10. Smith, A., King, E., Hindley, N., Barnetson, L., Barton, J. \& Kim, A.
The experience of research participation and the value of diagnosis in dementia: implications for practice. Journal of Mental Health 1998; 7 : 309-321

11. Farre, M., Lamas, X. \& Cami, J. Sensation seeking amongst healthy volunteers participating in phase I clinical trials. British Journal of Clinical Pharmacology 1995; 39: 405-409

12. Harrison, D. A. Volunteer motivation and attendance decisions: competitive theory testing in multiple samples from a homeless shelter. Journal of Applied Psychology1995; 80: 371-385

13. Ygge, B-M. \& Arnetz J.E. A study of non-response in a questionnaire survey of parents' views of paediatric care. Journal of Nursing Management, 2004, 12, 5-12

14. Pace, C., Grady, C., \& Emanuel E.J. What we don't know about informed consent? 2003. www.scidev.net

15. Bernard H: Social Research Methods: Qualitative and Quantitative Approaches. London, UK: Sage Publications; 2000

16. Guiley, R. E. 1999. The Encyclopedia of Witches and Witchcraft, second edition, p. 26. New York: Facts on File

17. Mather, G.A. \& Nichols, L.A. 1993. Dictionary of Cults, Sects, Religions and the Occult, p.40. Michigan: Zondervan Publishing House

18. Kavinya T 2007, A deeper look, interview with Bridon Mbaya. Malawi Medical Journal Vol 19, issue 2 www.mmj.medcol.mw/adeeperlook20 07vol19.2.htm

19. Sapienza, J. N., et al., Community engagement in Epidemiological Research. 2007 May-Jun; 7(3):247-52. Ambulatory Pediatric Association. p. 252

\section{Report on the workshop Oversight in Malawi"}

The Centre for Bioethics in Eastern and Southern Africa (CEBESA) in collaboration with College of Medicine Research and Ethics Committee (COMREC), National Health Science Research Council (NHSRC), and Pharmacy Medicines and Poisons Board (PMPB) hosted a two day workshop on "Enhancing Clinical Trial Oversight in Malawi" on the 19th and 20th June at Nkopola Lodge in Mangochi. The workshop was funded by the European and Developing Counties Clinical Trials Partnership (EDCTP) through a grant on strengthening national capacities in ethical review and clinical trial monitoring. The aim of the workshop was a critical look at research oversight in Malawi particularly in light of the implementation of sections of the PMPB Board Act of 1988 related to clinical trials. The workshop brought together stakeholders in health research from various institutions including University of North Carolina, Malawi Liverpool Wellcome Trust, University of Mzuzu, Kamuzu College of Nursing, Centre for Reproductive Health, College of Medicine and Blantyre Malaria Project. Also present at the workshop was Medical Rights Watch a medical student led organization whose goal is to promote and protect the rights and responsibilities of patients, research participants and health practitioners in Malawi.

Professor Joseph Mfutso-Bengo, Director of CEBESA and organizer of the workshop welcomed all the participants. The workshop was opened by the Principal of the College of Medicine, Professor Broadhead and was followed by an oversight of COMREC by the Postgraduate Dean Professor Eric Borgstein. Paul Ndebele of CEBESA gave an

\section{"Enhancing Clinical Trial}

introductory presentation on regulatory oversight of clinical trials. Mr. Aaron Sosola, Deputy Registrar of PMPB, gave a perspective of the PMPB, the PMPB Board Act and the systems and procedures that have been put into place by the Board regarding clinical trial monitoring. The workshop gave an opportunity for the PMPB to update stakeholders on the introduction of the clinical trial monitoring program which requires all research institutions to register their clinical trials with the Board. It was made clear that ethics and review committees (COMREC, NHSRC) and drug regulatory agencies (PMPB) have separate and distinct areas of focus. However there was consensus that further discussions need to be held amongst the stakeholders to see how best to harmonize the PMPB clinical trial monitoring with other existing systems. Participants discussed important issues related to the conduct of clinical trials this included whether submission of clinical trials should be concurrent or consequential to PMPB and ERCs, how the PMPB's Clinical Trial Review Committee can function efficiently, how ERCs and PMPB can cooperate, how to update researchers on the new measures and prepare research sites for the new developments. Participants were given the opportunity to review and comment on PMPB documents and make recommendations on the documents.

At the end of the workshop participants were familiar with PMPB legislation for clinical trials, and the PMPB tools and procedures to be used for regulating research. This workshop sensitized stakeholders on the impending new measures and gave the opportunity to stimulate what is hoped to be 